\title{
Noninvasive Assessment of Advanced Fibrosis Based on Hepatic Volume in Patients with Nonalcoholic Fatty Liver Disease
}

\author{
Tatsuya Hayashi ${ }^{1,2, *}$, Satoshi Saitoh ${ }^{2,3,4}$, Kei Fukuzawa ${ }^{1,2}$, Yoshinori Tsuji ${ }^{1,2}$, Junji Takahashi ${ }^{1,2}$, Yusuke Kawamura $^{2,3}$, \\ Norio Akuta ${ }^{2,3}$, Masahiro Kobayashi ${ }^{2,3}$, Kenji Ikeda ${ }^{2,3}$, Takeshi Fujii ${ }^{2,5}$, Tosiaki Miyati ${ }^{6}$, and Hiromitsu Kumada ${ }^{2,3}$

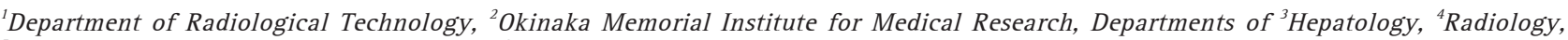 \\ ${ }^{5}$ Pathology, Toranomon Hospital, Tokyo, and ${ }^{6}$ Faculty of Health Sciences, Institute of Medical, Pharmaceutical and Health Sciences, Kanazawa \\ University, Kanazawa, Japan
}

See editorial on page 577.

Background/Aims: Noninvasive liver fibrosis evaluation was performed in patients with nonalcoholic fatty liver disease (NAFLD). We used a quantitative method based on the hepatic volume acquired from gadoxetate disodium-enhanced (Gd-EOB-DTPA-enhanced) magnetic resonance imaging (MRI) for diagnosing advanced fibrosis in patients with NAFLD. Methods: A total of 130 patients who were diagnosed with NAFLD and underwent Gd-EOB-DTPA-enhanced MRI were retrospectively included. Histological data were available for 118 patients. Hepatic volumetric parameters, including the left hepatic lobe to right hepatic lobe volume ratio (L/R ratio), were measured. The usefulness of the $L / R$ ratio for diagnosing fibrosis $\geq \mathrm{F} 3-4$ and F4 was assessed using the area under the receiver operating characteristic (AUROC) curve. Multiple regression analysis was performed to identify variables (age, body mass index, serum fibrosis markers, and histological features) that were associated with the L/R ratio. Results: The L/R ratio demonstrated good performance in differentiating advanced fibrosis (AUROC, 0.80; 95\% confidence interval, 0.72 to 0.88 ) from cirrhosis (AUROC, 0.87; $95 \%$ confidence interval, 0.75 to 0.99 ). Multiple regression analysis showed that only fibrosis was significantly associated with the L/R ratio (coefficient, 0.121; $p<0.0001$ ). Conclusions: The L/R ratio, which is not influenced by pathological parameters other than fibrosis, is useful for diagnosing cirrhosis in patients with NAFLD. (Gut Liver 2017;11:674683)
Key Words: Liver cirrhosis; Fibrosis; Gadoxetate disodium; Magnetic resonance imaging; Non-alcoholic fatty liver disease

\section{INTRODUCTION}

Nonalcoholic fatty liver disease (NAFLD) and nonalcoholic steatohepatitis (NASH) are common causes of liver disease in not only Western countries, but also in many Asian countries. ${ }^{1-3}$ The prevalence of suspected NAFLD has more than doubled over the past 20 years. ${ }^{4}$ Similar to viral hepatitis, NASH, which is a subcategory of NAFLD, can progress to fibrosis, cirrhosis, and hepatocellular carcinoma. ${ }^{5-7}$ In a systematic review, Argo et al. ${ }^{8}$ showed that 38\% of patients with NASH had progressive fibrosis over a mean follow-up period of 5.3 years. Angulo et al. ${ }^{9}$ reported that NASH progressed to cirrhosis in $10.8 \%$ of patients over a mean follow-up period of 15.6 years. Furthermore, Ascha et al. ${ }^{10}$ reported that $12.8 \%$ of NASH-cirrhotic patients developed hepatocellular carcinoma over a median follow-up period of 3.2 years. The gold standard for diagnosing fibrosis stage is biopsy; however, it is invasive and has drawbacks, such as sampling errors and intra- and inter-observer variabilities. ${ }^{11-14}$ Among all histological features, only fibrosis is associated with long-term outcomes in patients with NAFLD $;^{15}$ thus, diagnosing fibrosis stage is important. In patients with NAFLD, alternative noninvasive and quantitative diagnostic methods for liver fibrosis have been proposed.

Gadoxetate disodium (Gd-EOB-DTPA)-enhanced (Primovist; Bayer HealthCare, Osaka, Japan) is a new contrast agent that plays an important role in assessing chronic liver disease

Correspondence to: Tatsuya Hayashi

Department of Radiological Technology, Toranomon Hospital, 2-2-2 Toranomon, Minato-ku, Tokyo 105-8470, Japan

*Current Affiliation: Department of Medical Radiology, Faculty of Medical Technology, Teikyo University, 2-11-1, Kaga, Itabashi-ku, Tokyo 1738605, Japan

Tel: +81-90-1316-0672, Fax: +81-3-3560-7812,E-mail:ja_nei3@yahoo.co.jp

Received on September 3, 2016. Revised on December 14, 2016. Accepted on December 28, 2016. Published online June 27, 2017 pISSN 1976-2283 eISSN 2005-1212 https://doi.org/10.5009/gnl16440

@ This is an Open Access article distributed under the terms of the Creative Commons Attribution Non-Commercial License (http://creativecommons.org/licenses/by-nc/4.0) which permits unrestricted non-commercial use, distribution, and reproduction in any medium, provided the original work is properly cited. 
and detecting liver lesions. ${ }^{16-18}$ Apart from conventional dynamic images, hepatobiliary phase images are also available in magnetic resonance imaging (MRI) with Gd-EOB-DTPA. In Japan, dynamic contrast-enhanced MRI is recommended as an important surveillance and diagnostic tool for hepatocellular carcinoma in patients with chronic liver disease or cirrhosis. ${ }^{19}$ In fact, compared with multidetector computed tomography, Gd-EOB-DTPA-enhanced MRI is more useful for evaluating liver lesions; ${ }^{20,21}$ thus, we have had more opportunities to use it. Kawamura et al. ${ }^{22}$ reported that three-dimensional (3D)-MR images with Gd-EOB-DTPA in the hepatobiliary phase are useful for detecting advanced fibrosis in patients with NASH. They called 3D-MR images of the liver virtual MR laparoscopy and visually assessed them for irregularities of the liver surface, enlargement of the lateral segment, and atrophy of the right lobe. Ogura et al. ${ }^{23}$ evaluated fibrosis and reported that the method by Kawamura et al. ${ }^{22}$ was useful for evaluating liver fibrosis in patients with chronic hepatitis B and C viruses. However, this method is qualitative and subjective. In this study, we focused on quantifying the changes in liver volume (LV) due to atrophy of the right lobe or enlargement of the lateral segment from fibrosis using MR laparoscopy; we evaluated a novel quantitative method for diagnosing advanced fibrosis in patients with NAFLD using hepatic volume acquired from Gd-EOB-DTPAenhanced MRI.

\section{MATERIALS AND METHODS}

This retrospective study was approved by the Institutional Review Board of Toranomon Hospital (number: 1061), which waived the requirement for informed consent.

\section{Study population}

Consecutive patients who were diagnosed with NAFLD, including NASH, and who had undergone liver MRI with GdEOB-DTPA at our institution from October 2009 to March 2016 were included. The inclusion criteria were as follows: (1) history of daily alcohol intake <20 g/day; (2) negative for serum hepatitis C virus antibodies, hepatitis B surface antigen, antinuclear antibodies, and antimitochondrial antibodies, as determined by radioimmunoassay, enzyme-linked immunosorbent assay, or spot hybridization; (3) no underlying systemic autoimmune diseases, such as systemic lupus erythematosus and rheumatoid arthritis; (4) no underlying metabolic diseases, such as hemochromatosis, $\alpha$-1-antitrypsin deficiency, and Wilson disease; (5) no prior history of liver resection; and (6) ongoing Gd-EOBDTPA MRI within 1 year of histological examination (F0-4) or past diagnosis of fatty liver and the presence of liver nodularity and portal hypertension on MRI findings (F4). The inclusion criteria were fulfilled by 130 patients who underwent Gd-EOBDTPA MRI. The current study included (1) patients who were suspected of NASH and needed screening for hepatocellular carcinoma before liver biopsy $(n=128)$ and (2) those with NASH who were suspected of having lever lesions, as identified on ultrasonography $(n=2)$.

\section{Image acquisition for 3D-MRI}

All patients were intravenously administered Gd-EOB-DTPA at a weight-based dose of $0.025 \mathrm{mmol} / \mathrm{kg}(0.1 \mathrm{~mL} / \mathrm{kg}$; maximum volume $10 \mathrm{~mL}$ ), followed by $40 \mathrm{~mL}$ physiological saline; scanning in the hepatobiliary phase was performed 20 minutes after injecting Gd-EOB-DTPA. MRI was obtained with fat-saturated 3D T1-weighted gradient echo sequences using three systems: 1.5 T Magnetom Avanto (Siemens Healthcare, Erlangen, Germany); 1.5 T Excelart Vantage Power Plus (Toshiba Medical Systems, Tokyo, Japan); and 3.0 T Ingenia (Philips Healthcare, Best, The Netherlands). Sequence parameters were as follows: repetition time, 3.20 to 3.70 milliseconds; echo time, 1.30 to 1.46 milliseconds; slice thickness, 1.0 to $3.0 \mathrm{~mm}$; matrix (frequency), 256 to 336 ; matrix (phase), 192 to 256 ; flip angle, $10^{\circ}$ to $15^{\circ}$; band width, 450 to $783 \mathrm{~Hz} /$ pixel; parallel acceleration factor, 2 to 3; acquisition time, 17 to 25 seconds.

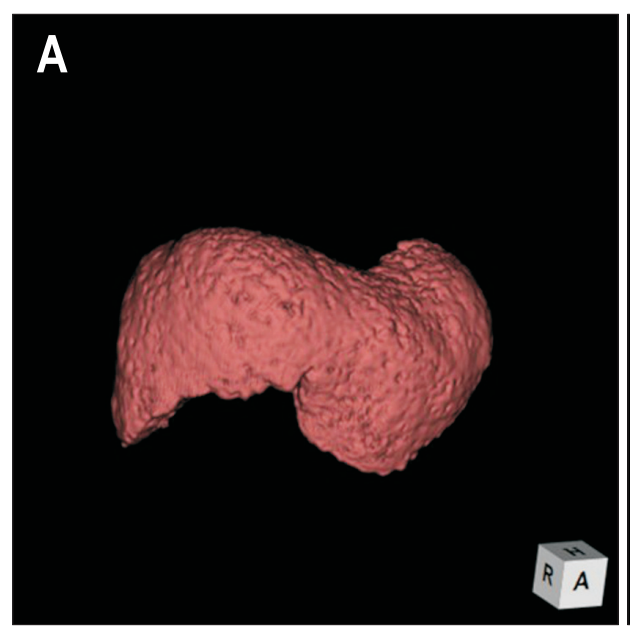

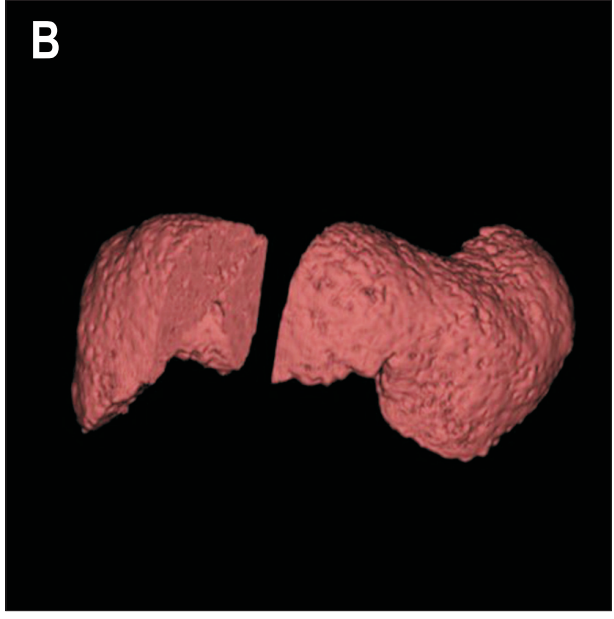

Fig. 1. Three-dimensional magnetic resonance images of a patient with fibrosis stage 4. (A) The entire liver and (B) the right and left lobes in a 67-year-old woman with fibrosis stage 4 are shown. Cantlie's line is used to divide the two lobes. The ratio of the left to right hepatic lobe volumes is 1.37 in this patient. 


\section{LV measurement using 3D-MRI}

Using Ziostation 2 (Ziosoft, Tokyo, Japan), LV measurement was performed by an MRI technologist who was blinded to the histological data. Furthermore, 3D image reconstructions of the liver were rendered from enhanced MRI data in the hepatobiliary phase (Fig. 1). On 3D images, various structures around the liver were manually marked and digitally removed. Care was taken to not exclude a part of the liver referring to axial, coronal, and sagittal liver images on a workstation. The intrahepatic vascular structures, such as portal veins and hepatic veins, were not included in LV because those structures were not enhanced in the hepatobiliary phase. Finally, the liver was extracted on the workstation, ${ }^{22,23}$ and hepatic volumes (total, right, and left) were measured. In addition, the ratio of left to right hepatic lobe volumes (L/R ratio) was calculated (Fig. 1B). Cantlie's line was used as the border between the right and left lobes of the liver and was drawn on the axial images so that the border line passed through the middle hepatic vein. The appearance of the 3D hepatic image is dependent on the pixel value threshold set. However, once the virtual hepatic 3D image has been reconstructed, the pixel value threshold does not influence hepatic volume measurement.

\section{Calculation of estimated LV}

To evaluate changes in LV with fibrosis progression, the ratio of the entire LV measured by MRI (MR-LV) to estimated LV (MR-LV/estimated LV), the ratio of the right hepatic lobe volume measured by MRI (MR-RLV) to estimated LV (MR-RLV) estimated LV), and the ratio of the left hepatic lobe volume measured by MRI (MR-LLV) to estimated LV (MR-LLV/estimated LV) were calculated. Body weight that was recorded at MR examination was used for calculating estimated LV. Estimated LV $\left(\mathrm{cm}^{3}\right)$ was calculated according to the formula of Zhou et al., ${ }^{24}$ 12.90xweight $(\mathrm{kg})+437.91$.

\section{Histological evaluation}

An expert pathologist who was blinded to the results of imaging and fibrosis markers evaluated the liver biopsy sections. Histological assessment was performed on the basis of the description of the pathology committee of the NASH Clinical Research Network. ${ }^{25}$ Steatosis was assessed visually on the basis of the percentage of hepatocytes affected and was graded as follows: $0(<5 \%$ ), 1 (mild, 5\% to 33\%), 2 (moderate, 34\% to 66\%), and 3 (severe, $>66 \%$ ). Lobular inflammation was graded on the basis of the number of inflammatory foci per $20 \times$ field as follows: grade 0,0 ; grade $1,<2$; grade 2,2 to 4 ; and grade 3, >4 Ballooning was graded as follows: grade 0 , none; grade 1 , few; or grade 2, many. Hepatic fibrosis was scored as follows: stage 0 , no fibrosis; stage 1, perisinusoidal or periportal fibrosis (1a, mild and perisinusoidal; 1b, moderate and perisinusoidal; 1c, portal); stage 2, perisinusoidal and periportal fibrosis without bridging; stage 3, bridging fibrosis; and stage 4, cirrhosis. In the analyses, fibrosis stages $1 \mathrm{a}, 1 \mathrm{~b}$, and $1 \mathrm{c}$ were treated as stage 1 . Hepatic iron content was evaluated using Perl's stain and was graded 0 to 3 .

\section{Serum fibrosis markers}

The aspartate aminotransferase to platelet ratio index (APRI) and BARD score were calculated to compare the diagnostic performance for fibrosis using volumetric parameters. The APRI score was calculated according to the following formula: APRI=aspartate aminotransferase (AST)/(AST upper limit of normal $) \times 100 /$ platelet count $\left(10^{9} / \mathrm{L}\right){ }^{26}$ The AST upper limit of normal was $33 \mathrm{IU} / \mathrm{L}$. The BARD score comprised three variables as follows: body mass index (BMI) $\geq 28 \mathrm{~kg} / \mathrm{m}^{2}, 1$ point; AST/ALT ratio $\geq 0.8$, 2 points; presence of diabetes, 1 point. $^{27}$

\section{Statistical analysis}

All analyses were performed using SPSS version 11.0.1 for Windows (SPSS Inc., Chicago, IL, USA) or Microsoft Excel 2013 (Microsoft Corp., Redmond, WA, USA). Associations between each hepatic volumetric parameter (MR-LV, MR-LV/estimated LV, MR-RLV/estimated LV, MR-LLV/estimated LV, and L/R ratios) and groups of different fibrosis stages were evaluated by Spearman rank correlation analysis. Receiver operating characteristic (ROC) analyses were performed to assess the diagnostic performance of the hepatic volumetric parameters and serum fibrosis markers as follows: (1) F0-2 versus F3-4 and (2) F0-3 versus F4. Sensitivity, specificity, accuracy, and area under the ROC (AUROC) were calculated; 95\% confidence intervals (95\% CI) were calculated for ROC analysis. AUROC values were considered excellent for 0.9 to 1.0 , good for 0.8 to 0.9 , moderate for 0.7 to 0.8 , and poor for $<0.7$. Univariate and multiple regression analyses were performed to assess the influence of age, BMI, serum fibrosis markers, and histopathological parameters, including steatosis, ballooning, inflammation, fibrosis, and iron content, on the $\mathrm{L} / \mathrm{R}$ ratio. A p-value of $<0.05$ was considered significant.

\section{RESULTS}

Of 130 patients, 118 were diagnosed with NAFLD on the basis of histological evaluation within a median interval of 6.5 days (interquartile range, 1 to 47 days) of Gd-EOB-DTPA MRI. The remaining 12 patients were clinically diagnosed with NASH cirrhosis, and all had esophageal varices. In this study population, liver MRI showed focal lesions ( $\mathrm{n}=26)$, hepatocellular carcinoma $(n=10)$, hemangioma $(n=8)$, liver cyst $(n=7)$, and focal nodular hyperplasia $(\mathrm{n}=1)$, all of which were $<20 \mathrm{~mm}$ in diameter. All patients in this study were classified into Child-Pugh class A. The characteristics of the 130 patients are shown in Table 1; there were 76 men and 54 women, and their median age was 59 years (interquartile range, 45 to 66 years). The distribution of fi- 
Table 1. Characteristics of Patients with Nonalcoholic Fatty Liver Disease $(n=130)$

\begin{tabular}{|c|c|}
\hline Variable & Value \\
\hline Male sex & $76(58.5)$ \\
\hline Age, yr & $59.0(45-66)$ \\
\hline Body mass index, $\mathrm{kg} / \mathrm{m}^{2}$ & $26.2(23.9-28.8)$ \\
\hline$>25$ & $80(61.5)$ \\
\hline$>30$ & $24(18.5)$ \\
\hline Diabetes mellitus & $29(22.3)$ \\
\hline High blood pressure & $16(12.3)$ \\
\hline Albumin, g/dL & $4.0(3.7-4.2)$ \\
\hline Total bilirubin, mg/dL & $0.9(0.7-1.1)$ \\
\hline Aspartate aminotransferase, IU/L & $42(31-64)$ \\
\hline Alanine aminotransferase, IU/L & $58(34-88)$ \\
\hline Platelet count, $\times 10^{3} / \mu \mathrm{L}$ & $196(153-245)$ \\
\hline$\gamma$-Glutamyltranspeptidase, IU/L & $61(36-95)$ \\
\hline Total cholesterol, mg/dL & $191(165-218)$ \\
\hline Triglyceride, mg/dL & $129(97-184)$ \\
\hline LDL-C, mg/dL & $105(81-133)$ \\
\hline HDL-C, mg/dL & $45(36-53)$ \\
\hline Ferritin, ng/mL & $246(141-401)$ \\
\hline Time interval between histological & $6.5(1-47)$ \\
\hline evaluation and MRI, day & \\
\hline \multicolumn{2}{|l|}{ Histological data } \\
\hline \multicolumn{2}{|l|}{ Steatosis grade $(\mathrm{n}=118)^{*}$} \\
\hline 0 & $1(0.9)$ \\
\hline 1 & $39(33.1)$ \\
\hline 2 & $50(42.4)$ \\
\hline 3 & $28(23.7)$ \\
\hline \multicolumn{2}{|l|}{ Hepatocellular ballooning $(\mathrm{n}=118)^{*}$} \\
\hline 0 & $10(8.5)$ \\
\hline 1 & $66(55.9)$ \\
\hline 2 & $42(35.6)$ \\
\hline \multicolumn{2}{|l|}{ Lobular inflammation $(\mathrm{n}=118)^{*}$} \\
\hline 0 & $3(2.5)$ \\
\hline 1 & 66 (55.9) \\
\hline 2 & 42 (37.3) \\
\hline 3 & $5(4.2)$ \\
\hline \multicolumn{2}{|l|}{ Stage of fibrosis $(n=130)^{\dagger}$} \\
\hline 0 & $5(3.9)$ \\
\hline $1 \mathrm{a}, \mathrm{b}, \mathrm{c}$ & $53(40.8)$ \\
\hline 2 & $15(11.5)$ \\
\hline 3 & $36(27.7)$ \\
\hline 4 & $21(17.8)$ \\
\hline \multicolumn{2}{|l|}{ Grade of iron $(n=118)^{*}$} \\
\hline 0 & $48(40.7)$ \\
\hline 1 & $57(48.3)$ \\
\hline 2 & $13(11.0)$ \\
\hline 3 & 0 \\
\hline
\end{tabular}

Data are presented as number (\%) or median (interquartile range). LDL-C, low density lipoprotein cholesterol; HDL-C, high density lipoprotein cholesterol; MRI, magnetic resonance imaging.

*Patients without histological diagnosis $(\mathrm{n}=12)$ were excluded from this item; ${ }^{\dagger}$ Fibrosis stage 4 includes patients who were clinically diagnosed with nonalcoholic steatohepatitis cirrhosis $(n=12)$. brosis stage was as follows: F0, 3.9\% (5/130); F1, 40.8\% (53/130); F2, 11.5\% (15/130); F3, 27.7\% (36/130); and F4, 17.8\% (21/130) (Table 1).

The volumetric parameters of the different fibrosis stages are shown in Table 2 and Fig. 2; among these, the MR-RLV/ estimated LV, MR-LLV/estimated LV, and L/R ratios demonstrated significant correlations with the fibrosis stage ( $\rho=-0.376$, $\mathrm{p}<0.0001 ; \rho=0.272, \mathrm{p}=0.002$; and $\rho=0.693, \mathrm{p}<0.0001$; respectively). The $\mathrm{L} / \mathrm{R}$ ratio was shown to increase as the severity of fibrosis progressed, with medians of 0.54, 0.57, 0.66, 0.75, and 1.04 for F0, F1, F2, F3, and F4, respectively. The fibrosis stage did not correlate significantly with other parameters, such as MR-LV and MR-LV/estimated LV ratio ( $\rho=-0.114, p=0.198$ and $\rho=-0.068, p=0.445$ ) (Table 2).

The diagnostic values of the hepatic volumetric parameters and serum fibrosis markers for advanced fibrosis (F3-4) and cirrhosis (F4) are shown in Table 3 and Fig. 3. The MR-LLV/ estimated LV ratio for advanced liver fibrosis and cirrhosis had poor diagnostic performance (AUROC, 0.66 and 0.60, respectively). The MR-RLV/estimated LV ratio exhibited poor capability for the prediction of advanced fibrosis (AUROC, 0.68; 95\% CI, 0.59 to 0.78 ) but good for the prediction of cirrhosis (AUROC, $0.80 ; 95 \% \mathrm{CI}, 0.68$ to 0.92 ). The $\mathrm{L} / \mathrm{R}$ ratio demonstrated good capability for discriminating advanced fibrosis (AUROC, 0.80; 95\% CI, 0.72 to 0.88) and cirrhosis (AUROC, 0.87; 95\% CI, 0.75 to 0.99 ). The optimal cutoff values of the $L / R$ ratio were 0.66 for $\geq F 3$ and 0.82 for $F 4$. The sensitivity and specificity of the L/R ratio for $\geq \mathrm{F} 3$ were 0.70 (95\% CI, 0.57 to 0.82 ) and 0.78 (95\% CI, 0.67 to 0.87 ), respectively, and for F4 were 0.86 (95\% CI, 0.64 to 0.97 ) and 0.91 (95\% CI, 0.84 to 0.96), respectively. APRI demonstrated good capability for discriminating advanced fibrosis (AUROC, 0.82; 95\% CI, 0.74 to 0.89) and moderate capability for discriminating cirrhosis (AUROC, 0.77; 95\% CI, 0.68 to 0.86). The BARD score exhibited moderate diagnostic performance for advanced liver fibrosis (AUROC, 0.75; 95\% CI, 0.67 to 0.83) and cirrhosis (AUROC, 0.75; 95\% CI, 0.66 to 0.85) (Table 3).

The $L / R$ ratio was positively correlated with age $(\rho=0.221$, $p=0.016)$, APRI $(\rho=0.323, p<0.001)$, BARD score $(\rho=0.237$, $\mathrm{p}=0.001)$, ballooning $(\rho=0.191, p=0.038)$, inflammation $(\rho=0.324, p<0.001)$, and fibrosis $(\rho=0.547, p<0.0001)$ (Table 4). On multiple regression analysis, only fibrosis was significantly associated with the $\mathrm{L} / \mathrm{R}$ ratio (coefficient, $0.121 ; \mathrm{p}<0.0001$ ) (Table 4).

\section{DISCUSSION}

The relationships of various volumetric parameters of the liver with fibrosis progression were evaluated. Of these parameters, the $\mathrm{L} / \mathrm{R}$ ratio increased with fibrosis progression and was not dependent on other histological features such as steatosis, ballooning, inflammation, and iron. Furthermore, the ratio was useful to diagnose cirrhosis in patients with NAFLD. Angulo 
Table 2. Association of Hepatic Volumetric Parameters with Fibrosis Stage

\begin{tabular}{|c|c|c|c|c|c|c|c|}
\hline Parameter & F0 & $\mathrm{F} 1$ & $\mathrm{~F} 2$ & F3 & $\mathrm{F} 4$ & $\rho^{*}$ & p-value \\
\hline \multirow[t]{2}{*}{ MR-LV, $\mathrm{cm}^{3}$} & $1,391.0$ & $1,653.6$ & $1,611.4$ & $1,704.5$ & $1,463.8$ & -0.114 & 0.198 \\
\hline & $(1,301.4-1,588.9)$ & $(1,500.7-2,025.8)$ & $(1,343.7-1,761.7)$ & $(1,518.8-1,982.4)$ & (994.9-1,701.6) & & \\
\hline MR-LV/estimated LV ratio & $1.10(1.07-1.15)$ & $1.25(1.12-1.45)$ & $1.24(1.10-1.30)$ & $1.26(1.11-1.43)$ & $1.12(0.87-1.39)$ & -0.068 & 0.445 \\
\hline MR-RLV/estimated LV ratio & $0.70(0.65-0.79)$ & $0.80(0.72-0.92)$ & $0.76(0.67-0.79)$ & $0.73(0.64-0.84)$ & $0.57(0.38-0.74)$ & -0.376 & $<0.0001$ \\
\hline $\mathrm{L} / \mathrm{R}$ ratio & $0.54(0.52-0.63)$ & $0.57(0.48-0.63)$ & $0.66(0.57-0.72)$ & $0.75(0.63-0.86)$ & $1.04(0.87-1.23)$ & 0.693 & $<0.0001$ \\
\hline
\end{tabular}

Data are presented as median (interquartile range).

MR-LV, liver volume (LV) measured by magnetic resonance imaging (MRI); MR-LV/estimated LV ratio, ratio of MR-LV to estimated liver volume; MR-RLV, right hepatic lobe volume measured by MRI; MR-RLV/estimated LV ratio, ratio of MR-RLV to estimated liver volume; MR-LLV, left hepatic lobe volume measured by MRI; MR-LLV/estimated LV ratio, ratio of MR-LLV to estimated liver volume; L/R ratio, ratio of left to right hepatic lobe volume.

*All data were tested using Spearman rank correlation test.
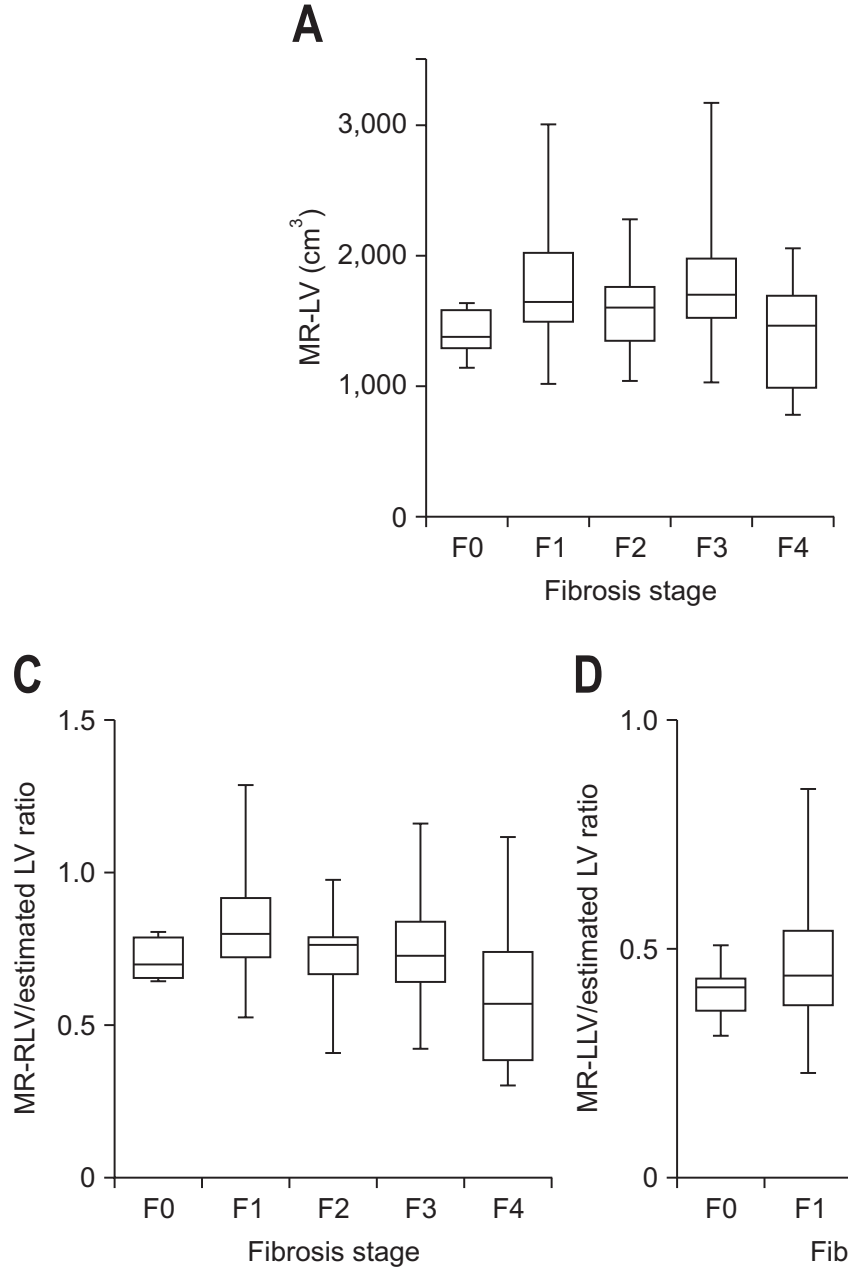

D
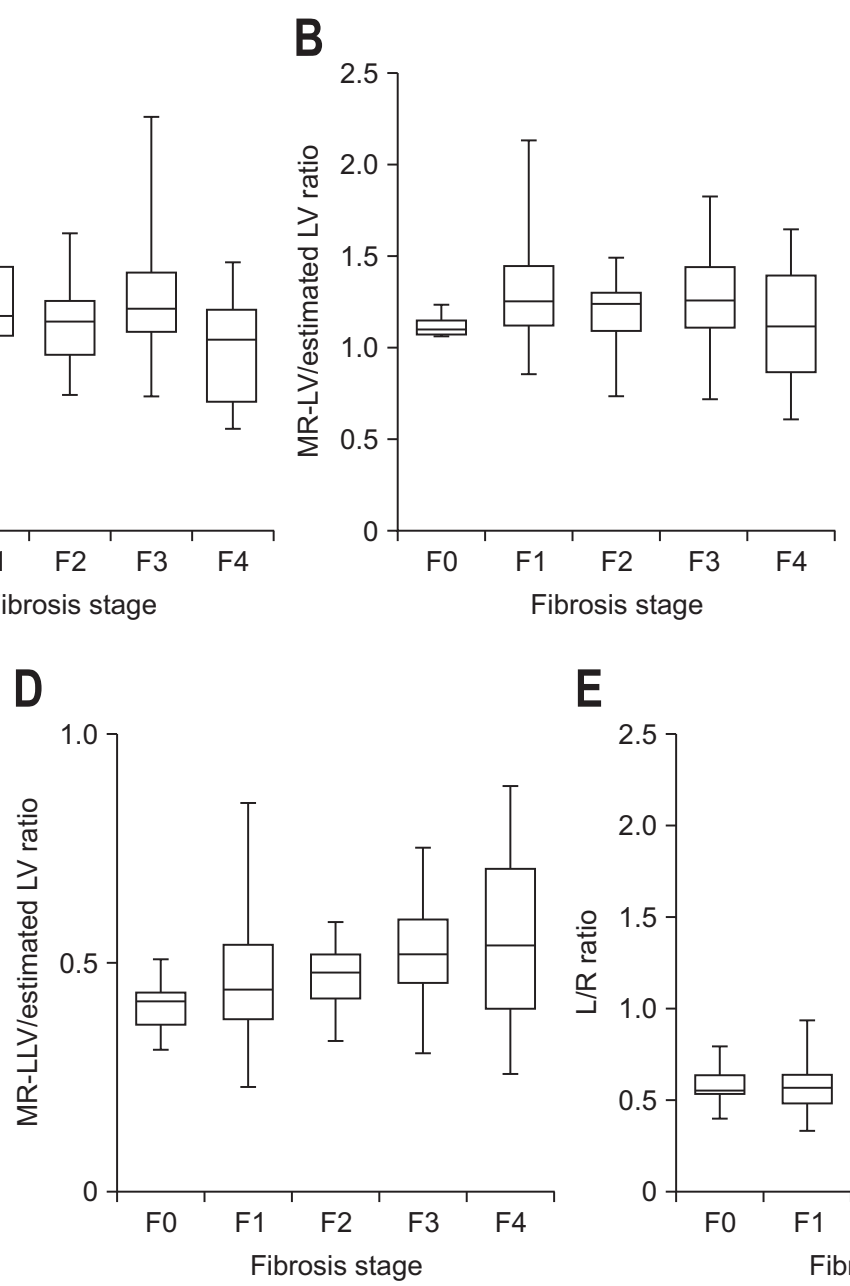

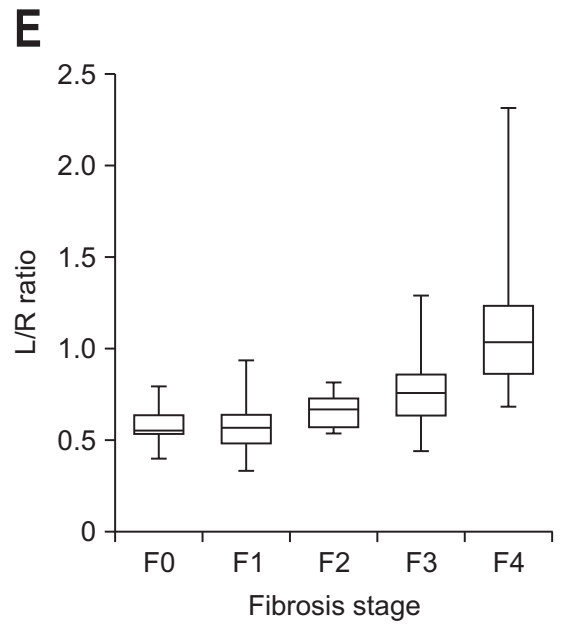

Fig. 2. Relationship of liver volumetric parameters with the fibrosis stage. The parameters measured are (A) MR-LV, (B) MR-LV/estimated LV, (C) MR-RLV/estimated LV, (D) MR-LLV/estimated LV, and (E) L/R ratios. Boxes represent interquartile ranges, which are demarcated by median values. Error bars represent minimum and maximum values. The MR-RLV/estimated LV, MR-LLV/estimated LV, and L/R ratios demonstrated a significant correlation with the fibrosis stage ( $\rho=-0.376, p<0.0001 ; \rho=0.272, p=0.002$; and $\rho=0.693, p<0.0001$, respectively).

MR-LV/estimated LV, ratio of entire liver volume (LV) measured by magnetic resonance imaging (MRI) to estimated LV; MR-RLV/estimated LV, ratio of right hepatic lobe volume measure by MRI to estimated liver volume; MR-LLV/estimated LV, ratio of left hepatic lobe volume measure by MRI to estimated liver volume; $\mathrm{L} / \mathrm{R}$, ratio of left to right hepatic lobe volumes. 
Table 3. Receiver Operating Characteristic Curve Analysis of the Ability of Hepatic Volumetric Methods and Serum Fibrosis Markers to Diagnose the Fibrosis Stage

\begin{tabular}{|c|c|c|}
\hline Parameter & $\leq \mathrm{F} 2$ vs $\geq \mathrm{F} 3$ & $\leq \mathrm{F} 3$ vs $\mathrm{F} 4$ \\
\hline $\begin{array}{l}\text { MR-RLV/estimated } \\
\text { LV ratio cutoff }\end{array}$ & 0.76 & 0.67 \\
\hline Accuracy & $0.65(0.56-0.73)$ & $0.75(0.66-0.82$ \\
\hline Sensitivity & $0.70(0.57-0.82)$ & $0.71(0.48-0.89$ \\
\hline Specificity & $0.60(0.48-0.72)$ & $0.75(0.66-0.83$ \\
\hline PPV & $0.58(0.45-0.70)$ & $0.36(0.22-0.52$ \\
\hline NPV & $0.72(0.59-0.83)$ & $0.93(0.86-0.97$ \\
\hline AUROC & $0.68(0.59-0.78)$ & $0.80(0.68-0.92$ \\
\hline $\begin{array}{l}\text { MR-LLV/estimated } \\
\text { LV ratio cutoff }\end{array}$ & 0.51 & 0.53 \\
\hline Accuracy & $0.65(0.56-0.73)$ & $0.55(0.46-0.64$ \\
\hline Sensitivity & $0.61(0.48-0.74)$ & $0.52(0.30-0.74$ \\
\hline Specificity & $0.67(0.55-0.78)$ & $0.56(0.46-0.65$ \\
\hline PPV & $0.59(0.46-0.72)$ & $0.19(0.10-0.31$ \\
\hline NPV & $0.69(0.57-0.79)$ & $0.86(0.76-0.93$ \\
\hline AUROC & $0.66(0.56-0.76)$ & $0.60(0.43-0.76$ \\
\hline $\mathrm{L} / \mathrm{R}$ ratio cutoff & 0.66 & 0.82 \\
\hline Accuracy & $0.75(0.66-0.82)$ & $0.90(0.84-0.95$ \\
\hline Sensitivity & $0.70(0.57-0.82)$ & $0.86(0.64-0.97$ \\
\hline Specificity & $0.78(0.67-0.87)$ & $0.91(0.84-0.96$ \\
\hline PPV & $0.71(0.58-0.83)$ & $0.64(0.44-0.81)$ \\
\hline NPV & $0.77(0.66-0.86)$ & $0.97(0.92-0.99$ \\
\hline AUROC & $0.80(0.72-0.88)$ & $0.87(0.75-0.99$ \\
\hline APRI cutoff & 0.90 & 0.93 \\
\hline Accuracy & $0.78(0.70-0.85)$ & $0.69(0.61-0.77$ \\
\hline Sensitivity & $0.72(0.58-0.83)$ & $0.71(0.48-0.89$ \\
\hline Specificity & $0.84(0.73-0.91)$ & $0.69(0.59-0.77$ \\
\hline PPV & $0.77(0.64-0.88)$ & $0.31(0.18-0.45$ \\
\hline NPV & $0.79(0.68-0.88)$ & $0.93(0.85-0.97$ \\
\hline AUROC & $0.82(0.74-0.89)$ & $0.77(0.68-0.86$ \\
\hline BARD score cutoff & 2 & 2 \\
\hline Accuracy & $0.69(0.61-0.77)$ & $0.62(0.53-0.70$ \\
\hline Sensitivity & $0.75(0.62-0.86)$ & $0.95(0.76-1.00$ \\
\hline Specificity & $0.64(0.52-0.75)$ & $0.55(0.45-0.65$ \\
\hline PPV & $0.62(0.50-0.74)$ & $0.29(0.19-0.41)$ \\
\hline NPV & $0.77(0.65-0.87)$ & $0.98(0.91-1.00$ \\
\hline AUROC & $0.75(0.67-0.83)$ & $0.75(0.66-0.85)$ \\
\hline
\end{tabular}

Data in parentheses are 95\% confidence intervals. Receiver operating characteristic analyses were performed.

MR-RLV, right hepatic lobe volume measured by magnetic resonance imaging (MRI); LV, liver volume; PPV, positive predictive value; NPV, negative predictive value; AUROC, area under the receiver operating characteristic curve; MR-LLV, left hepatic lobe volume measured by MRI; L/R ratio, ratio of left to right hepatic lobe volume; APRI, aspartate aminotransferase to platelet ratio index.
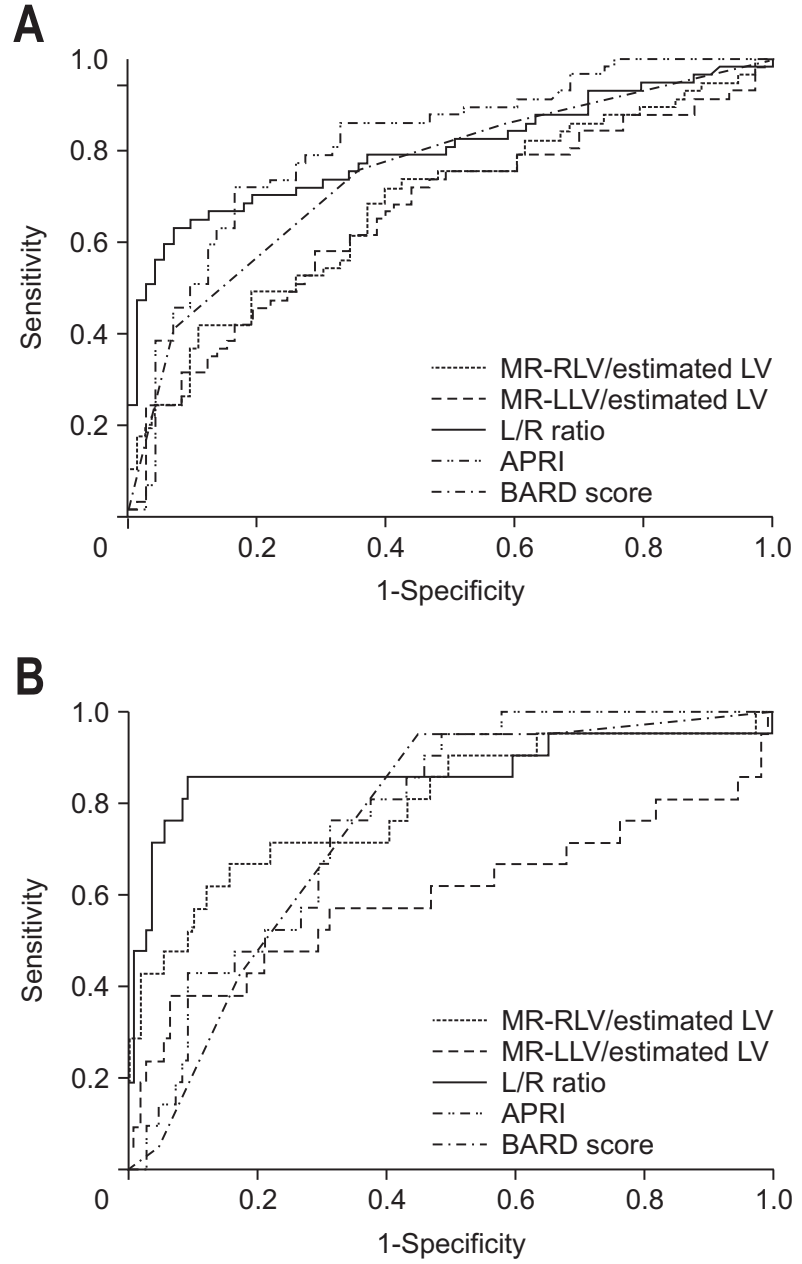

Fig. 3. Receiver operating characteristic curves for hepatic volumetric parameters and serum fibrosis markers for the diagnosis of fibrosis stage (A) $\mathrm{F} \geq 3$ and (B) F4.

MR-RLV/estimated LV, ratio of right hepatic lobe volume measured by magnetic resonance imaging (MRI) to estimated liver volume (LV); MR-LLV/estimated LV, ratio of left hepatic lobe volume measured by MRI to estimated liver volume; $L / R$, ratio of left to right hepatic lobe volumes; APRI, aspartate aminotransferase to platelet ratio index.

et al. ${ }^{15}$ reported that hazard ratios (HRs) for the association of death or liver transplantation with each fibrosis stage compared with F0 were: F1, HR 1.88, 95\% CI 1.28 to 2.77; F2, HR 2.89, 95\% CI 1.93 to 4.33; F3, HR 3.76, 95\% CI 2.40 to 5.89; and F4, HR 10.9, 95\% CI 6.06 to 19.62. In particular, the risk was high for cirrhosis. Based on their results, diagnosing cirrhosis was more important for understanding the long-term outcomes of patients with NAFLD. Therefore, our findings are important because the $\mathrm{L} / \mathrm{R}$ ratio, which is not influenced by pathological parameters other than fibrosis, is useful for diagnosing cirrhosis in patients with NAFLD.

Although the cause of enlargement of the lateral segment and atrophy of the right lobe in cirrhosis is unclear (differences in nutrition or hormones supplied to the lobes are thought to be among the pathophysiologic mechanisms), the phenomenon 
Table 4. Influence of Age, BMI, Serum Fibrosis Markers, and Histopathological Parameters on the L/R Ratio in Patients with NAFLD (n=118)

\begin{tabular}{|c|c|c|c|c|c|c|}
\hline \multirow{2}{*}{ Independent variable } & \multicolumn{2}{|c|}{ Univariate analysis } & \multicolumn{4}{|c|}{ Multiple regression analysis } \\
\hline & $\rho^{*}$ & p-value & Coefficient & SE & t-value & p-value \\
\hline Age & 0.221 & 0.016 & -0.001 & 0.002 & -0.796 & 0.428 \\
\hline BMI & 0.104 & 0.263 & -0.006 & 0.004 & -1.274 & 0.205 \\
\hline APRI & 0.323 & $<0.001$ & 0.017 & 0.022 & 0.792 & 0.430 \\
\hline BARD score & 0.237 & 0.001 & 0.014 & 0.014 & 0.958 & 0.340 \\
\hline Steatosis & 0.020 & 0.828 & 0.010 & 0.022 & 0.462 & 0.645 \\
\hline Ballooning & 0.191 & 0.038 & -0.043 & 0.030 & -1.440 & 0.153 \\
\hline Inflammation & 0.324 & $<0.001$ & -0.007 & 0.030 & -0.233 & 0.816 \\
\hline Fibrosis & 0.547 & $<0.0001$ & 0.121 & 0.019 & 6.54 & $<0.0001$ \\
\hline Iron & -0.133 & 0.150 & -0.010 & 0.024 & -0.438 & 0.662 \\
\hline
\end{tabular}

In multiple regression analysis, only fibrosis was significantly associated with the L/R ratio.

BMI, body mass index; L/R ratio, ratio of left to right hepatic lobe volume; NAFLD, nonalcoholic fatty liver disease; APRI, aspartate aminotransferase to platelet ratio index.

*Spearman rank correlation coefficient.

is well known. ${ }^{28}$ Kawamura et al..$^{22}$ used 3D-MRI as virtual MR laparoscopy to visually assess the irregularities of the liver surface, enlargement of the lateral segment, and atrophy of the right lobe for diagnosing advanced fibrosis in patients with NASH. Their method demonstrated high sensitivity (100\%), specificity (90\%), positive predictive value (82\%), and negative predictive value (100\%). However, their method was qualitative and subjective. In addition, the differentiation between F3 and F4 was not assessed in their study. Ogura et al. ${ }^{23}$ applied MR laparoscopy to patients with viral hepatitis B and C and confirmed the same hepatic morphological changes in patients with cirrhosis; however, they did not perform a quantitative assessment. ${ }^{23}$ The objective and quantitative diagnosis of fibrosis progression was useful, and the present study adds new quantitative information regarding the diagnosis of cirrhosis with the use of the $\mathrm{L} / \mathrm{R}$ ratio. Although the present method demonstrated a moderate diagnostic performance for $\geq F$ 3, it demonstrated a high diagnostic performance for F4 (cirrhosis). Therefore, a combination of visual assessment and the present quantitative method may be useful to diagnose advanced fibrosis (F3-4) and distinguish F4 from F3. This point requires further study. The MR-RLV/estimated LV ratio also exhibited good capability for diagnosing cirrhosis, whereas the MR-LLV/estimated LV ratio exhibited poor capability for cirrhosis; however, this ratio showed a significant positive correlation with the fibrosis stage. These ratios were inferior to the $L / R$ ratio for the quantitative diagnosis of fibrosis because the $L / R$ ratio used the ratio of the opposite hepatic morphological changes (enlargement of the left hepatic lobe and atrophy of the right hepatic lobe), which enhanced the diagnostic performance of the $\mathrm{L} / \mathrm{R}$ ratio. Conversely, the opposite hepatic morphological changes may negate the influence of the entire hepatic volume in patients with advanced fibrosis. Such factors could make it difficult to assess the entire hepatic volume as fibrosis progresses.
Some fibrosis markers have been established for liver diseases. APRI is a simple serum fibrosis marker, and is widely available. APRI was originally developed as a hepatic fibrosis marker for patients with chronic hepatitis C. ${ }^{26}$ However, the reproducibility of AST levels or platelet count measurement was questionable, and APRI had larger variability. ${ }^{29-31}$ Recently, APRI has been used in patients with NAFLD. Several studies have evaluated the diagnostic performance of APRI for diagnosing fibrosis stage in patients with NAFLD (AUROC for severe fibrosis [F3-4], 0.568 to 0.850 ; AUROC for cirrhosis [F4], 0.786 to 0.842). ${ }^{32-36} \mathrm{In}$ the present study, the ability of APRI to diagnose F3-4 and F4 was similar to that reported in those studies (AUROC for severe fibrosis, 0.82; AUROC for cirrhosis, 0.77). The BARD score was also used as a simple serum fibrosis marker. ${ }^{27}$ This score can be calculated using widely available parameters (BMI, AST/ALT ratio, and presence of diabetes). The utility of the score was investigated in patients with NAFLD (AUROC for severe fibrosis [F3-4], 0.67 to 0.81; AUROC for cirrhosis [F4], 0.62). ${ }^{27,37-39}$ The main limitation of the BARD score is that it is likely to exhibit high false positive results. ${ }^{39}$ Patients with a BARD score of $\geq 2$ are predicted to have severe fibrosis, if they have a BMI of $\geq 28$ $\mathrm{kg} / \mathrm{m}^{2}$ and presence of diabetes despite having a normal level of AST/ALT ratio. ${ }^{39}$ In the present study, in terms of diagnosing cirrhosis, low positive predictive value (0.29) was observed with the BARD score. Thus, results of the present study demonstrated that the $\mathrm{L} / \mathrm{R}$ ratio could be more advantageous than APRI and BARD scores for diagnosing cirrhosis in patients with NAFLD, although the diagnostic performance of the $\mathrm{L} / \mathrm{R}$ ratio was similar to that of APRI for severe fibrosis.

Recently, the usefulness of elastography, including ultrasound elastography and MR elastography, for assessing liver fibrosis was reported. FibroScan is a typical method among the ultrasound elastography methods. Cassinotto et al ${ }^{40}$ reported that using FibroScan, liver stiffness was positively correlated with 
the fibrosis stage $(\rho=0.67)$ and had good capability for diagnosing advanced fibrosis (AUROC, 0.86) and cirrhosis (AUROC, 0.87). However, liver stiffness measured with widely used $\mathrm{M}$ probe was overestimated in patients with large skin capsular distance. ${ }^{41}$ An XL probe has been developed for such obese patients. ${ }^{42}$ However, it is not widely available. In addition, our MR volumetric method was not affected by the subcutaneous fat, and the L/R ratio exhibited high diagnostic performance for cirrhosis similar to that of ultrasound elastography. Kim et al. ${ }^{43}$ reported the use of MR elastography for fibrosis staging in patients with NASH, with an AUROC of 0.954 for detecting advanced fibrosis (F3-4); however, they did not evaluate the diagnosis of cirrhosis. In another study, Loomba et al. ${ }^{44}$ showed that the AUROC for MR elastography discriminated advanced fibrosis (F3-4) from F0-2 and cirrhosis from F0-3 fibrosis (0.924 and 0.894, respectively). However, MR elastography has several limitations. The method cannot evaluate the stiffness of the whole liver. The technique estimates the stiffness from only a part of the liver. In addition, the left hepatic lobe is avoided in the measurement because of cardiac motion artifact. ${ }^{45}$ Furthermore, MR elastography is not easy to perform on a routine basis because it requires special equipment and additional scan time. In contrast, by the present method, we could evaluate the volume data from the whole liver for the diagnosis of advanced fibrosis. In addition, although the volumetric method can be widely used without needing a highly specialized MRI machine, extra equipment, or expensive analysis software, it showed high diagnostic performance, particularly in cirrhosis (AUROC, 0.87), at the same level as MR elastography. Furthermore, the present method may be a practical adjunct to routine MRI enhanced by the contrast agent Gd-EOB-DTPA, which enables acquisition of both hepatobiliary phase and standard dynamic images to detect and characterize liver lesions. ${ }^{20,46,47}$ This is important, because advanced fibrosis is an important risk factor for hepatocellular carcinoma, and hepatocellular carcinoma is a major cause of mortality in patients with NASH having advanced fibrosis. ${ }^{48}$

The present study had several limitations. First, the method was not applicable for patients who underwent hepatectomy or those with a severe decrease in kidney function. Nevertheless, MRI with Gd-EOB-DTPA is repeatable and practical for patients with advanced age or those with liver dysfunction. No patient with severely impaired liver function was found in this study. The liver often shows low signal intensity in such patients on hepatobiliary phase imagery. However, although 3D construction by this method is more time consuming in these patients, it is not too difficult because complicated segmentation is not needed. The volumetric method generally requires less than 5 minutes for 3D reconstruction and analysis. Second, advanced cirrhosis or burnt-out NASH, in which the characteristic features of NASH were reported to disappear, was not assessed. ${ }^{49}$ Further study is required to evaluate the applicability of the present method to these states. Third, in the present study, the MRI pa- rameters varied because multivendor MRI equipment was used. A previous study showed that there was no significant difference between the MR-LV acquired using a 2-mm slice and the LVs acquired using 4-, 6-, and 8-mm slices, although the measurement method was not exactly the same as that used in the present study. ${ }^{50}$ In another study of the brain, no significant difference was observed in the volume of the hippocampus using different thicknesses ( 1 to $5 \mathrm{~mm}$ ). ${ }^{51}$ Thus, in the present study, the slice thicknesses (1 to $3 \mathrm{~mm}$ ) did not affect the LV measurements. In addition, the axial resolutions used in our preliminary study did not substantially interfere with the MR-LV ratio. Last, the study was a retrospective single-center study; therefore, the possibility of selection bias could not be avoided.

In conclusion, the $\mathrm{L} / \mathrm{R}$ ratio was significantly correlated with fibrosis stage. In patients with NAFLD, although the L/R ratio exhibited capability similar to that of APRI for diagnosing advanced fibrosis, this parameter may be useful for diagnosing cirrhosis without histological features other than fibrosis. Furthermore, the method is widely available and does not require special equipment.

\section{CONFLICTS OF INTEREST}

H.K. has received honoraria and expenses for speaking at events organized by MSD K.K., Bristol-Myers Squibb, GlaxoSmithKline K.K., and Janssen Pharmaceutical K.K. K.I. has received honoraria and expenses for speaking at events organized by Sumitomo Dainippon Pharma Co., Ltd., Eisai Co., Ltd., and Olympus Corp. All other authors report no conflicts of interest.

\section{ACKNOWLEDGEMENTS}

The authors would like to thank Naomi Tagaya, Mariko Hiramoto, Yasutaka Tanuma, and Takahisa Tokimori for their help with this study.

\section{REFERENCES}

1. Angulo P. Nonalcoholic fatty liver disease. N Engl J Med 2002; 346:1221-1231.

2. Rinella ME. Nonalcoholic fatty liver disease: a systematic review. JAMA 2015;313:2263-2273.

3. Fan JG, Farrell GC. Epidemiology of non-alcoholic fatty liver disease in China. J Hepatol 2009;50:204-210.

4. Welsh JA, Karpen S, Vos MB. Increasing prevalence of nonalcoholic fatty liver disease among United States adolescents, 19881994 to 2007-2010. J Pediatr 2013;162:496-500.e1.

5. Adams LA, Lymp JF, St Sauver J, et al. The natural history of nonalcoholic fatty liver disease: a population-based cohort study. Gastroenterology 2005;129:113-121.

6. Ratziu V, Bonyhay L, Di Martino V, et al. Survival, liver failure, and hepatocellular carcinoma in obesity-related cryptogenic cir- 
rhosis. Hepatology 2002;35:1485-1493.

7. Kawamura Y, Arase Y, Ikeda K, et al. Large-scale long-term follow-up study of Japanese patients with non-alcoholic fatty liver disease for the onset of hepatocellular carcinoma. Am J Gastroenterol 2012;107:253-261.

8. Argo CK, Northup PG, Al-Osaimi AM, Caldwell SH. Systematic review of risk factors for fibrosis progression in non-alcoholic steatohepatitis. J Hepatol 2009;51:371-379.

9. Angulo P. Long-term mortality in nonalcoholic fatty liver disease: is liver histology of any prognostic significance? Hepatology 2010;51:373-375.

10. Ascha MS, Hanouneh IA, Lopez R, Tamimi TA, Feldstein AF, Zein NN. The incidence and risk factors of hepatocellular carcinoma in patients with nonalcoholic steatohepatitis. Hepatology 2010;51:1972-1978.

11. Piccinino F, Sagnelli E, Pasquale G, Giusti G. Complications following percutaneous liver biopsy: a multicentre retrospective study on 68,276 biopsies. J Hepatol 1986;2:165-173.

12. Regev A, Berho M, Jeffers LJ, et al. Sampling error and intraobserver variation in liver biopsy in patients with chronic HCV infection. Am J Gastroenterol 2002;97:2614-2618.

13. Colloredo G, Guido M, Sonzogni A, Leandro G. Impact of liver biopsy size on histological evaluation of chronic viral hepatitis: the smaller the sample, the milder the disease. J Hepatol 2003;39:239244.

14. Ratziu V, Charlotte F, Heurtier A, et al. Sampling variability of liver biopsy in nonalcoholic fatty liver disease. Gastroenterology 2005;128:1898-1906.

15. Angulo P, Kleiner DE, Dam-Larsen S, et al. Liver fibrosis, but no other histologic features, is associated with long-term outcomes of patients with nonalcoholic fatty liver disease. Gastroenterology 2015;149:389-397.e10.

16. Frydrychowicz A, Lubner MG, Brown JJ, et al. Hepatobiliary MR imaging with gadolinium-based contrast agents. J Magn Reson Imaging 2012;35:492-511.

17. Bashir MR, Gupta RT, Davenport MS, et al. Hepatocellular carcinoma in a North American population: does hepatobiliary MR imaging with Gd-EOB-DTPA improve sensitivity and confidence for diagnosis? J Magn Reson Imaging 2013;37:398-406.

18. Higaki A, Ito K, Tamada T, et al. Prognosis of small hepatocellular nodules detected only at the hepatobiliary phase of Gd-EOB-DTPA-enhanced MR imaging as hypointensity in cirrhosis or chronic hepatitis. Eur Radiol 2014;24:2476-2481.

19. Kudo M, Matsui O, Izumi N, et al. JSH consensus-based clinical practice guidelines for the management of hepatocellular carcinoma: 2014 update by the Liver Cancer Study Group of Japan. Liver Cancer 2014;3:458-468.

20. Sano K, Ichikawa T, Motosugi U, et al. Imaging study of early hepatocellular carcinoma: usefulness of gadoxetic acid-enhanced MR imaging. Radiology 2011;261:834-844.

21. Böttcher J, Hansch A, Pfeil A, et al. Detection and classification of different liver lesions: comparison of Gd-EOB-DTPA-enhanced
MRI versus multiphasic spiral CT in a clinical single centre investigation. Eur J Radiol 2013;82:1860-1869.

22. Kawamura Y, Saitoh S, Arase Y, et al. Three-dimensional magnetic resonance imaging for stringent diagnosis of advanced fibrosis associated with nonalcoholic steatohepatitis. Hepatol Int 2013;7:850-858.

23. Ogura S, Saitoh S, Kawamura Y, et al. Magnetic resonance laparoscopy: a new non-invasive technique for the assessment of chronic viral liver disease. Hepatol Res 2013;43:836-845.

24. Zhou XP, Lu T, Wei YG, Chen XZ. Liver volume variation in patients with virus-induced cirrhosis: findings on MDCT. AJR Am J Roentgenol 2007;189:W153-W159.

25. Kleiner DE, Brunt EM, Van Natta M, et al. Design and validation of a histological scoring system for nonalcoholic fatty liver disease. Hepatology 2005;41:1313-1321.

26. Wai CT, Greenson JK, Fontana RJ, et al. A simple noninvasive index can predict both significant fibrosis and cirrhosis in patients with chronic hepatitis C. Hepatology 2003;38:518-526.

27. Harrison SA, Oliver D, Arnold HL, Gogia S, Neuschwander-Tetri BA. Development and validation of a simple NAFLD clinical scoring system for identifying patients without advanced disease. Gut 2008;57:1441-1447.

28. Tan KC. The right posterior hepatic notch sign. Radiology 2008;248:317-318.

29. Piton A, Poynard T, Imbert-Bismut F, et al. Factors associated with serum alanine transaminase activity in healthy subjects: consequences for the definition of normal values, for selection of blood donors, and for patients with chronic hepatitis C. MULTIVIRC group. Hepatology 1998;27:1213-1219.

30. Leroy V, Halfon P, Bacq Y, et al. Diagnostic accuracy, reproducibility and robustness of fibrosis blood tests in chronic hepatitis $\mathrm{C}$ : a meta-analysis with individual data. Clin Biochem 2008;41:13681376.

31. Castera L. Non-invasive assessment of liver fibrosis in chronic hepatitis C. Hepatol Int 2011;5:625-634.

32. Loaeza-del-Castillo A, Paz-Pineda F, Oviedo-Cárdenas E, SánchezAvila F, Vargas-Vorácková F. AST to platelet ratio index (APRI) for the noninvasive evaluation of liver fibrosis. Ann Hepatol 2008;7:350-357.

33. Calès P, Lainé F, Boursier J, et al. Comparison of blood tests for liver fibrosis specific or not to NAFLD. J Hepatol 2009;50:165-173.

34. Fujii H, Enomoto M, Fukushima W, et al. Noninvasive laboratory tests proposed for predicting cirrhosis in patients with chronic hepatitis $\mathrm{C}$ are also useful in patients with non-alcoholic steatohepatitis. J Gastroenterol 2009;44:608-614.

35. Kruger FC, Daniels CR, Kidd M, et al. APRI: a simple bedside marker for advanced fibrosis that can avoid liver biopsy in patients with NAFLD/NASH. S Afr Med J 2011;101:477-480.

36. Subasi CF, Aykut UE, Yilmaz Y. Comparison of noninvasive scores for the detection of advanced fibrosis in patients with nonalcoholic fatty liver disease. Eur J Gastroenterol Hepatol 2015;27:137141. 
37. Wong VW, Vergniol J, Wong GL, et al. Diagnosis of fibrosis and cirrhosis using liver stiffness measurement in nonalcoholic fatty liver disease. Hepatology 2010;51:454-462.

38. Ruffillo G, Fassio E, Alvarez E, et al. Comparison of NAFLD fibrosis score and BARD score in predicting fibrosis in nonalcoholic fatty liver disease. J Hepatol 2011;54:160-163.

39. Lee TH, Han SH, Yang JD, Kim D, Ahmed M. Prediction of advanced fibrosis in nonalcoholic fatty liver disease: an enhanced model of BARD score. Gut Liver 2013;7:323-328.

40. Cassinotto C, Boursier J, de Lédinghen V, et al. Liver stiffness in nonalcoholic fatty liver disease: a comparison of supersonic shear imaging, FibroScan, and ARFI with liver biopsy. Hepatology 2016;63:1817-1827.

41. Shen F, Zheng RD, Shi JP, et al. Impact of skin capsular distance on the performance of controlled attenuation parameter in patients with chronic liver disease. Liver Int 2015;35:2392-2400.

42. Kumagai E, Korenaga K, Korenaga M, et al. Appropriate use of virtual touch quantification and FibroScan M and XL probes according to the skin capsular distance. J Gastroenterol 2016;51:496505.

43. Kim D, Kim WR, Talwalkar JA, Kim HJ, Ehman RL. Advanced fibrosis in nonalcoholic fatty liver disease: noninvasive assessment with MR elastography. Radiology 2013;268:411-419.

44. Loomba R, Wolfson T, Ang B, et al. Magnetic resonance elastography predicts advanced fibrosis in patients with nonalcoholic fatty liver disease: a prospective study. Hepatology 2014;60:1920-1928.

45. Venkatesh SK, Yin M, Ehman RL. Magnetic resonance elastography of liver: clinical applications. J Comput Assist Tomogr 2013;37:887-896.

46. Saito K, Kotake F, Ito N, et al. Gd-EOB-DTPA enhanced MRI for hepatocellular carcinoma: quantitative evaluation of tumor enhancement in hepatobiliary phase. Magn Reson Med Sci 2005;4:1-9.

47. Golfieri R, Renzulli M, Lucidi V, Corcioni B, Trevisani F, Bolondi L. Contribution of the hepatobiliary phase of Gd-EOB-DTPAenhanced MRI to Dynamic MRI in the detection of hypovascular small $(\leq 2 \mathrm{~cm})$ HCC in cirrhosis. Eur Radiol 2011;21:1233-1242.

48. Hashimoto E, Yatsuji S, Tobari M, et al. Hepatocellular carcinoma in patients with nonalcoholic steatohepatitis. J Gastroenterol 2009;44 Suppl 19:89-95.

49. Yoshioka Y, Hashimoto E, Yatsuji S, et al. Nonalcoholic steatohepatitis: cirrhosis, hepatocellular carcinoma, and burnt-out NASH. J Gastroenterol 2004;39:1215-1218.

50. Reiner CS, Karlo C, Petrowsky H, Marincek B, Weishaupt D, Frauenfelder T. Preoperative liver volumetry: how does the slice thickness influence the multidetector computed tomography- and magnetic resonance-liver volume measurements? J Comput Assist Tomogr 2009;33:390-397.

51. Laakso MP, Juottonen K, Partanen K, Vainio P, Soininen H. MRI volumetry of the hippocampus: the effect of slice thickness on volume formation. Magn Reson Imaging 1997;15:263-265. 